\title{
Imaging mass in three dimensions
}

David E. Wittman, Vera Margoniner, J. Anthony Tyson, Judith G. Cohen, Andrew Becker, et al.

David E. Wittman, Vera Margoniner, J. Anthony Tyson, Judith G. Cohen, Andrew Becker, lan P. Dell'Antonio, "Imaging mass in three dimensions," Proc. SPIE 4836, Survey and Other Telescope Technologies and Discoveries, (24 December 2002); doi: 10.1117/12.457372

Event: Astronomical Telescopes and Instrumentation, 2002, Waikoloa, Hawai'i, United States 


\title{
Imaging mass in three dimensions
}

\author{
D. Wittman ${ }^{a}$, V. E. Margoniner ${ }^{a}$, J. A. Tyson ${ }^{a}$, J. G. Cohen ${ }^{b}$, A. C. Becker ${ }^{a}$, \\ I. P. Dell'Antonio ${ }^{c}$ \\ ${ }^{a}$ Bell Labs, Lucent Technologies; ${ }^{b}$ Caltech; ${ }^{c}$ Brown University
}

\begin{abstract}
We explore a possible "killer app" for the LSST and similar surveys: imaging mass in three dimensions. We describe its scientific importance, practical techiques for realizing it, the current state of the art and how it might scale to the LSST.
\end{abstract}

Keywords: surveys, gravitational lensing, tomography

\section{INTRODUCTION}

LSST is a proposed $\sim 8 \mathrm{~m}$ telescope with a $3^{\circ}$ field of view, capable of repeated deep imaging over all the sky accessible from its site (Tyson et al. 2002; Angel et al. 2001; see also these proceedings). It has many science drivers, from detecting potentially hazardous asteroids to studying large-scale structure in the universe. Here we describe an application, imaging mass in three dimensions, which is just emerging as a feasible science and may take on great importance by the time LSST is operational.

One of the most fundamental ways to test our understanding of cosmology is to examine the evolution of structure, or clustering, in the universe. We have detailed observations of the structure at one early epoch, $z \sim$ 1100 , from anisotropies in the cosmic microwave background (CMB). It is thought that these inhomogeneities grow through gravitational collapse to become the much larger inhomogeneities we see today in the form of galaxy clusters, filaments, and voids. If we understand the nature of dark matter and of gravity, and know the values of global parameters such as $\Omega_{m}$, the mean density of matter, we should be able to predict the clustering at all epochs subsequent to that of the CMB. This would be quite a strict end-to-end test if we had good observations of structure at many epochs; a model with a wrong value of a global parameter or a false picture of the nature of dark matter could match some epochs, but not all.

Unfortunately, most of the matter in the universe does not emit light, so unbiased measurements of structure are sorely lacking in the epochs between the CMB and the present. If we really want to test the foundations of the model, we cannot assume that the structure seen in the galaxy distribution accurately represents the mass distribution. Instead, we must use gravitational means to detect mass, luminous or not. Redshift surveys offer one way, because they measure not only galaxy positions but also velocities, which are influenced by inhomogeneities in the mass distribution. Redshift surveys of the local universe (to $z \sim 0.3$ ) such as the SDSS and the 2dFGRS are currently yielding reasonable constraints on structure. However, redshift surveys are limited to the local universe because acquiring redshifts takes so much telescope time.

A better way to measure earlier epochs is offered by weak gravitational lensing, in which the "cosmic wallpaper" of distant galaxies is distorted by intervening mass distributions before reaching us (Figure 1). We shall see that lensing has a broad redshift sensitivity, peaking at $z \sim 0.5-1$, which is a nice complement to the CMB and to nearby redshift surveys. Such redshifts can also be easily probed by detecting clusters of galaxies (rather than individual galaxies) through X-ray or optical emmision, or through the Sunyaev-Zeldovich effect. In contrast to these methods, lensing does not depend on the dynamical state or baryonic content of the cluster, nor does it require even a cluster to probe these redshifts.

Lensing is sensitive to all the mass projected between a source and observer. The difficulty of estimating redshifts of faint sources has led to the practice of lumping all sources together, producing a single two-dimensional map of the projected mass distribution. The emergence in recent years of photometric redshift techniques (Connolly et al. 1995, Hogg et al. 1998) opens the possibility of separating sources according to redshift, thus allowing us to probe along the line of sight as well. Because looking at more distant structures is equivalent to looking back in time, this offers a look at the evolution of structure, which is precisely what is needed to complement current information on structure at very early and very late epochs. 


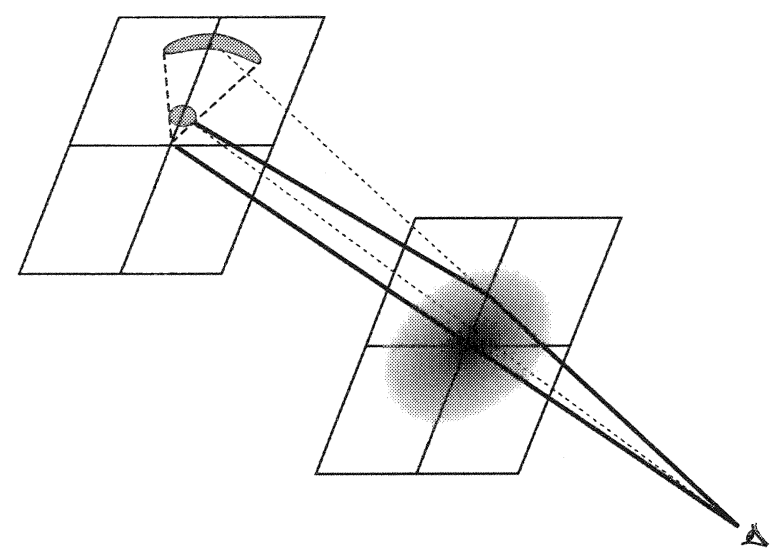

Figure 1. Schematic of gravitational lensing. The source is a distant galaxy which happens to be circular in this example (circle in plane at left). Were the lens not intervening, light would take a straight path and the observer would see its true shape. With the lens intervening, the rays are bent and the image seen by the observer is displaced and distorted tangentially (blue arc). In reality, nearly collinear systems giving such a strong distortions are extremely rare. Instead, weak lensing examines thousands of sources far off-axis for a statistical tendency to be oriented tangentially. If these sources could be binned by distance from the observer, a three-dimensional view of the intervening mass distribution could be built up.

\section{LENSING BASICS}

Figure 1 shows the basic idea of gravitational lensing. Because space-time is bent by mass, bundles of light rays passing by the mass are distorted before reaching the observer. The strength of this effect depends on a combination of the angular diameter distances from observer to source, from observer to lens, and from lens to source called the distance ratio.

The left panel of Figure 2 shows the run of distance ratio with source redshift for a few fixed lens redshifts, and the right panels shows the run of distance ratio with lens redshift for a few fixed source redshifts. For a given lens, the effect increases monotonically as the source gets further behind the lens. We will use this fact to determine the location of the lens the line of sight. Furthermore, for a typical source redshift $z \sim 1$, structures at $z \sim 0.5$ are most effectively probed, nicely complementing the CMB and local probes.

To date, most lensing work consists of following up on already-known clusters with known redshifts. The only distance-related variable is then the source redshift distribution. Ignorance of this distribution is encapsulated into a single parameter, the mean distance ratio, and one- or two-dimensional quantities such as projected mass profiles or distributions are measured. The question of the lens location along the line of sight is sidestepped because is is assumed that the lens is coincident with the cluster of known redshift. This will not be good enough for large surveys like LSST, for several reasons.

First, there may be dark clusters which are incapable of yielding spectroscopic redshifts. There are already reports in the literature of such dark clusters (Erben et al. 2000; Gray et al. 2001a; Umetsu \& Futamase 2000; Miralles et al. 2002). Lensing without photometric redshifts is sufficient to find these, but without a lens redshift, the most basic physical parameters of such clusters, such as mass and mass-to-light ratio must remain unknown. We don't even know how dark they are!

Second, such reports of dark clusters are received with some skepticism by the astrophysical community. Skeptics may be convinced if the shear from a putative dark cluster shows the predicted increase with source redshift (and vanishes for source redshift less than the lens redshift). It is one thing to show that all sources collectively show some distortion indicative of a lensing effect, and quite another to show that when divided into independent subsamples, each subsample behaves as expected.

Finally, large surveys like LSST may discover so many clusters that spectroscopic followup is impractical even if none are dark. This technique cannot completely substitute for spectroscopic redshifts because of its 

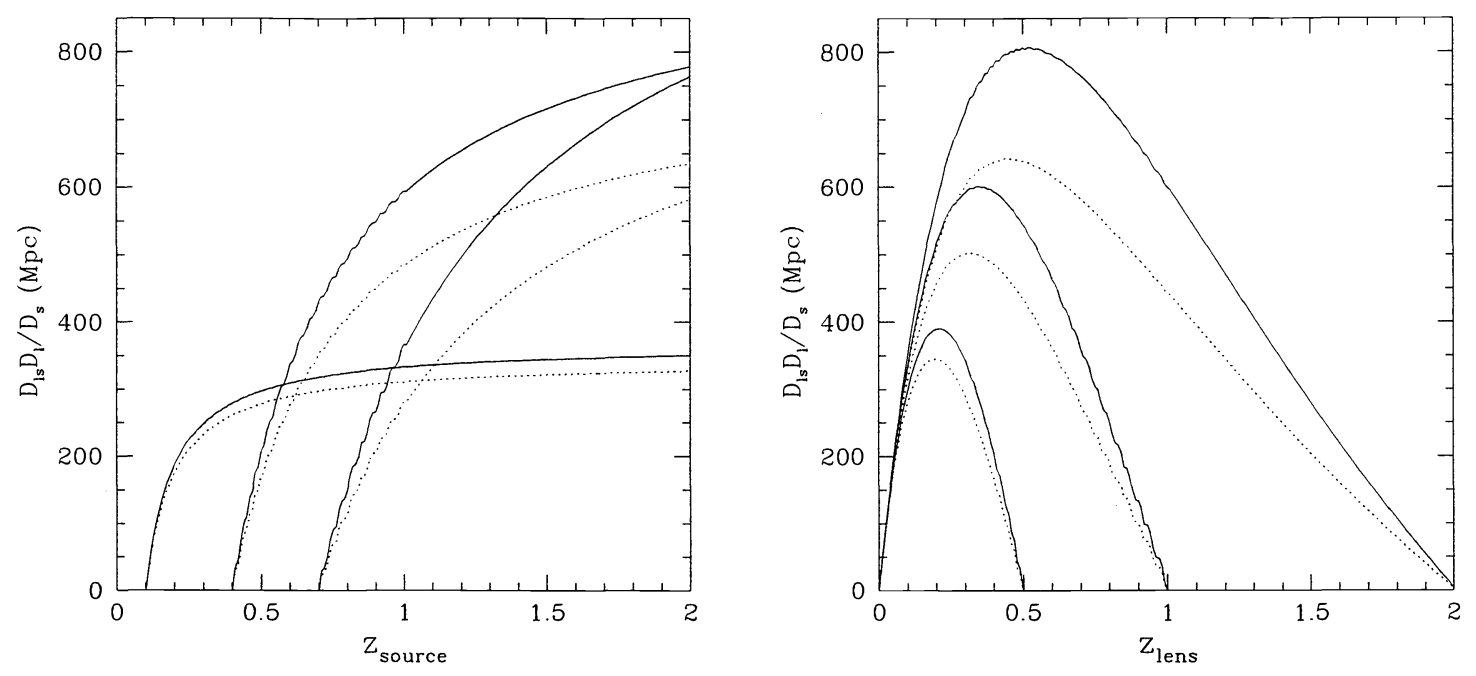

Figure 2. Left: distance ratio as a function of source redshift, for several lens redshifts (indicated by the intersections of the curves with the horizontal axis) and several cosmologies. The cosmologies are $\Lambda$-dominated (solid lines, $H_{0}=70$ $\mathrm{km} \mathrm{s}^{-1} \mathrm{Mpc}^{-1}, \Omega_{m}=0.3, \Omega_{\Lambda}=0.7$ ) and open (dashed lines, $H_{0}=70 \mathrm{~km} \mathrm{~s}^{-1} \mathrm{Mpc}^{-1}, \Omega_{m}=0.4, \Omega_{\Lambda}=0$ ). Right: same, but as a function of lens redshift, for several values of source redshift (which correspond to the right-hand end of each curve).

limited accuracy, but it may yield redshifts good enough for studying samples statistically. It may also be necessary to assign a rough redshift to winnow the interesting candidates for spectroscopy down to a reasonable number.

\section{PHOTOMETRIC REDSHIFTS}

Obtaining spectroscopic redshifts of faint objects is extremely difficult. Current deep redshift surveys (e.g. Cohen et al. 1999) go to $R=24$, yet weak lensing is routinely done on samples with $24<R<26$. Such faint samples are necessary in weak lensing, despite the lack of knowledge of their redshift distribution, for two reasons. First, we know at least that the mean redshift of the sample increases as the magnitude increases. We desire as high-redshift a sample as possible, because it allows us to study higher-redshift structures, and because the lensing effect of a fixed mass increases as the source redshift increases. Second, pushing ever deeper allows more sources to be used, which is critical because the statistical signal-to-noise goes as the square root of the number of sources.

Photometric redshifts now allow us to get some knowledge of source redshifts without doing spectroscopy. The basic idea is to take images through a series of filters to build up some knowledge about the observed spectral energy distribution (SED). There are two basic approaches to using this information. The so-called empirical approach uses a spectroscopically calibrated sample to derive an empirical mapping between colors and redshift, which can then be applied to any galaxy whose colors are known. The template-fitting approach is essentially Bayesian. Computationally redshifted template SEDs (e.g. elliptical, spiral, and irregular) are compared with the observed colors to derive a likelihood of the data given the model. Priors such as magnitude and size may be added at this stage (e.g. Benitez 2000), and then an integral over the template types ultimately produces a redshift likelihood distribution. Figure 3 illustrates this method.

Each approach has its strengths and weaknesses. The empirical approach is limited by the spectroscopic sample used to calibrate it. Estimating redshifts of $R \sim 26$ galaxies using an $R<24$ calibrated sample is asking for trouble, but it is fine for many applications which would not push beyond the spectroscopic limits. On the other hand, the template-fitting method is limited by the templates. The templates are usually synthetic SEDs 


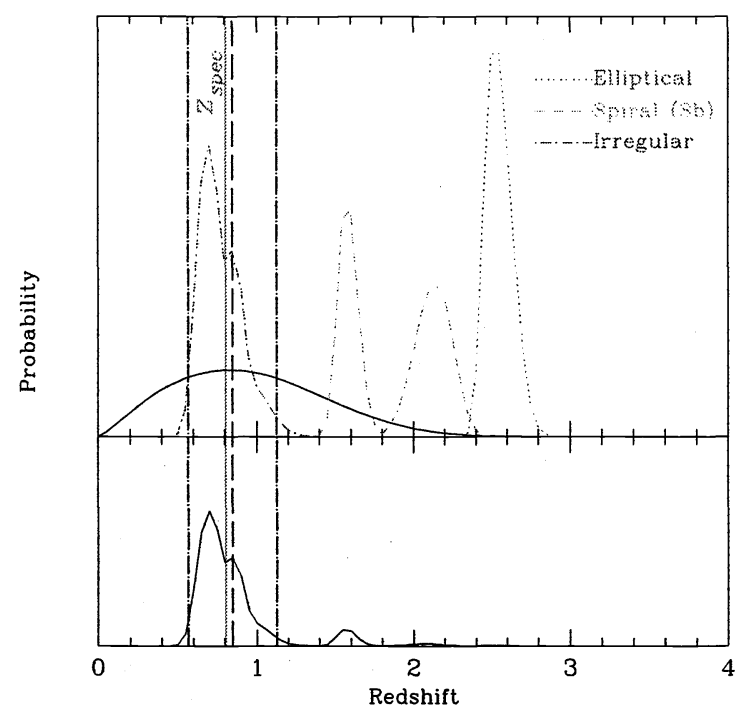

Figure 3. The top panel indicates two kinds of redshift probability distribution. The dotted, dashed, and dot-dash lines indicate the probability derived from the comparison between observed colors and the expected colors of an elliptical, Sb, and irregular galaxy at each redshift. The solid line indicates the redshift probability distribution based on the apparent magnitude in the $\mathrm{R}$ band. The lower panel shows the final redshift probability distribution. The spectroscopic redshift is indicated by a vertical solid line. The mean and $\mathrm{rms}$ of the photometric redshift probability distribution are indicated by vertical dashed lines.

which may or may not adequately mimic the variety of galaxies found in nature. Using SEDs measured from real galaxies only reintroduces the problems of the empirical method. With either method, it is desirable to have a spectroscopic sample to check the results. In the case of the empirical method, this sample must be independent of the fitted sample to give a fair assessment.

On balance, it seems that the template method offers the most promising way to assign photometric redshifts to galaxies unreachable with spectroscopy, as long as sufficient care goes into choosing the templates. Figure 4 illustrates the accuracy achievable today using a sample from the Caltech Faint Galaxy Redshift Survey (CFGRS, Cohen et al. 1999), using photometry from the Deep Lens Survey (DLS, Wittman et al. 2002a). The rms scatter is $18 \%$ in $1+z$. Importantly, there is very little bias: The mean photometric redshift of sample of galaxies at a given spectroscopic redshift differs from the true value by $\sim 3 \%$. The per-galaxy accuracy is limited mostly by the use of only four filters confined to CCD wavelengths $\left(B V R z^{\prime}\right)$; the same algorithm applied to the Hubble Deep Field with seven filters extending to $K$ yields about $10 \%$ accuracy per galaxy.

\section{LENSING WITH PHOTOMETRIC REDSHIFTS: A SIMPLE EXAMPLE}

The most straightforward application of photometric redshifts in lensing is to examine the shear about an already-identified lens as a function of source redshift. Wittman et al. (2001; hereafter W2001) were the first to do this. They had discovered their lens from its shear in a magnitude-selected sample, so its redshift was not immediately known. Followup spectroscopy of the cluster of galaxies around the lens showed it to be at $z=0.276$. Their shear versus source photometric redshift results are shown in Figure 5 . The data are consistent with a lens at $z=0.276$; the formal best fit is $z=0.30$.

This was the first time a lens redshift had been measured rather than assumed. Admittedly the measurement is not very precise: Figure 6 shows the lens redshift probability distribution. Also, W2001 was based on a single $40^{\prime}$ field, which will not yield a sample of more than about one cluster. Hence the importance of large surveys in extending this technique and exploring its behavior for a variety of lens redshifts, masses, and mass distributions. 

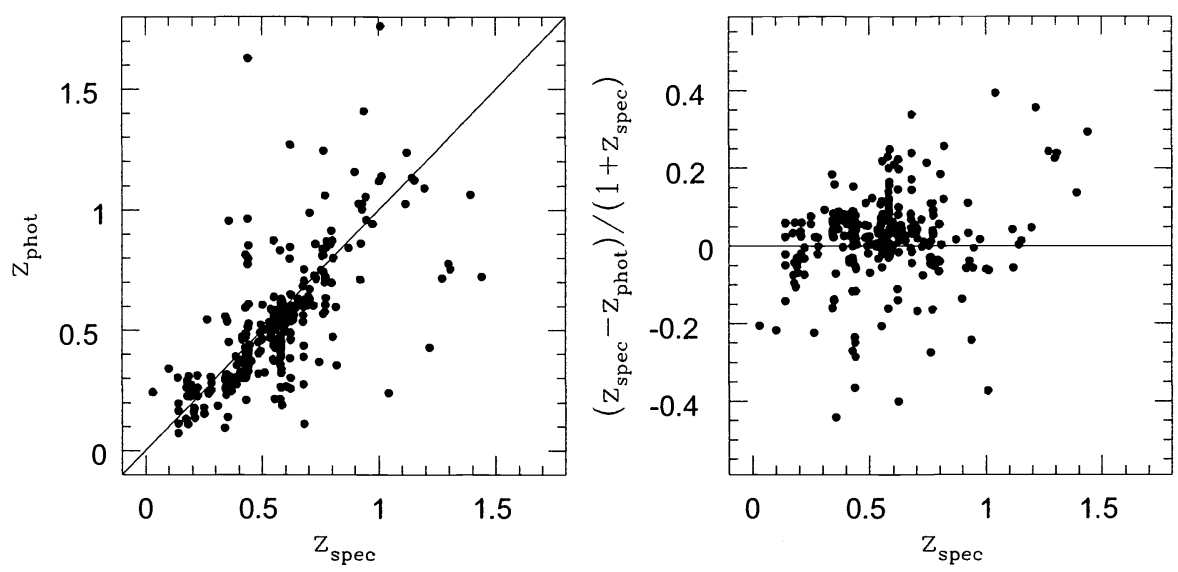

Figure 4. Typical photometric redshift performance with four filters not extending beyond $1 \mu \mathrm{m}$. Left: photometric versus spectroscopic redshift in a subfield of the Deep Lens Survey including hundreds of galaxies from the Caltech Faint Galaxy Redshift Survey. Right: residuals versus spectroscopic redshift. The photometric redshift algorithm was template-fitting with a magnitude prior, as illustrated in the previous figure.

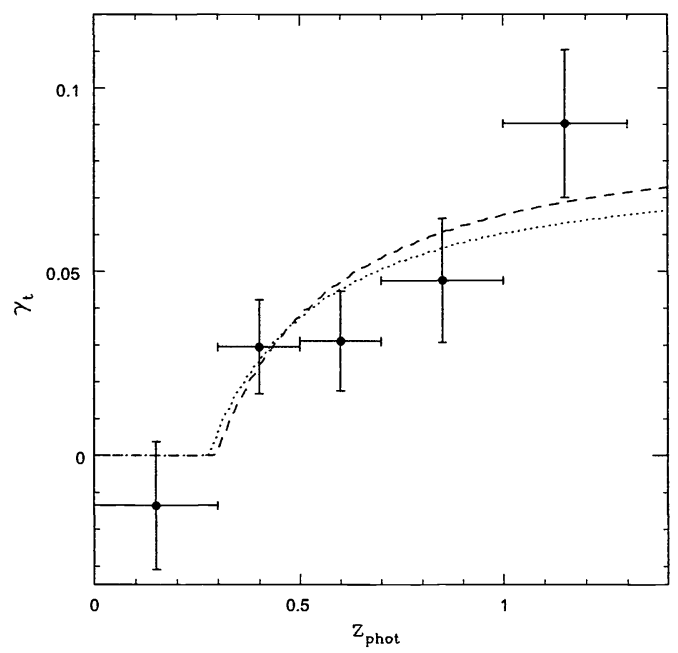

Figure 5. Shear around a galaxy cluster as a function of source photometric redshift. The dotted and dashed lines represent the shear expected from lenses at $z=0.276$ and $z=0.30$, the spectroscopic and shear-derived redshifts respectively. 


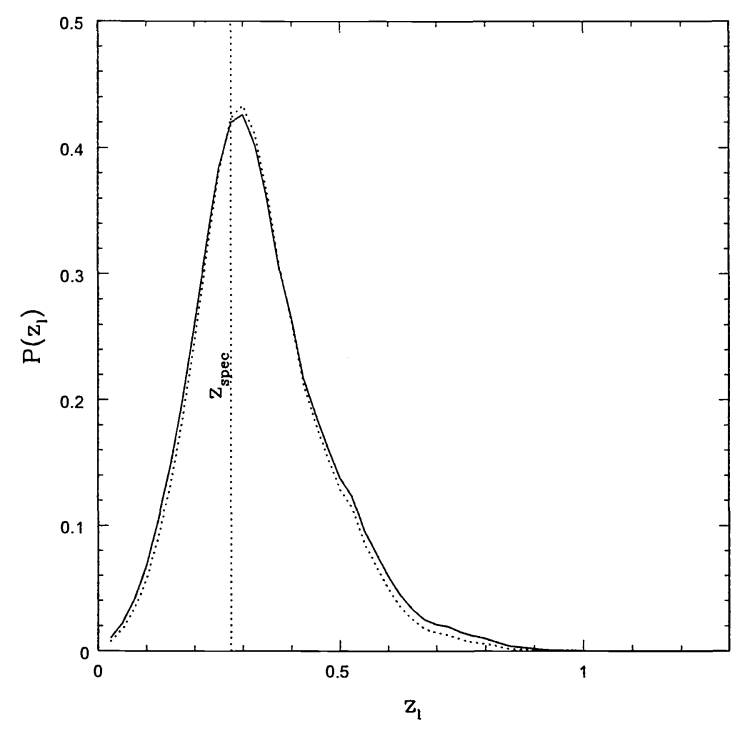

Figure 6. Redshift probability distribution for the W2001 lens, derived from the shear data alone. The solid and dotted lines correspond to two different assumptions about the cluster profile. Either assumption leads to a most probable lens redshift within 0.03 of the spectroscopic value.

The DLS is a survey currently taking $B V R z^{\prime}$ imaging of $28 \mathrm{deg}^{2}$ to about the same depth as the one small field in W2001. About 50-100 clusters are expected to be discovered over that area. We have already found one at a redshift of $z=0.68$, with tomography of similar quality to that shown in Figures 5 and 6 , in a field which is not yet to complete depth. This significantly extends the redshift range over which this tomographic technique is proven to work (Wittman et al. 2002b). The DLS has also found some lower-redshift clusters, some of which are identified with Abell clusters and most of which are new.

\section{LENSING WITH PHOTOMETRIC REDSHIFTS: OTHER TECHNIQUES}

Although the above example is a necessary first step, it is not really tomography. All information about the mass distribution in the plane of the sky has been collapsed into a single shear measurement so that its behavior in the third dimension can be brought out with reasonable signal-to-noise. We can do true tomography by making a series of two-dimensonal maps, each from a different source redshift bin. We did this for the cluster in W2001, with a low redshift bin showing no cluster and a higher redshift bin showing the cluster prominently (Figure 7).

The tradeoff here is that by making maps, we are trying to extract more information from the same dataset, compared to the simple shear versus redshift curve. Hence the maps will be noisier. To insure good S/N in the maps here, we limit ourselves to two redshift bins rather than the five in Figure 5. Already there is theoretical work on how to be economical with the tomography data by extracting only the important features (Taylor 2002; Hu \& Keeton 2002; Hu 2002). Nevertheless, we need LSST for its vast area coverage if we hope to do precision cosmology.

We can also measure the evolution of structure without reconstructing the actual mass distributions. One way to do this is with the shear correlation functions, or cosmic shear. Pairs of galaxies with a small projected separation on the sky will have their shapes correlated by lensing, regardless of whether a specific structure causing the correlation can be identified. The correlation becomes stronger for higher-redshift sources, because their light bundles pass through more structures together.

The first detections of this effect were published in 2000 (Wittman et al. 2000; van Waerbeke et al. 2000; Bacon, Refregier \& Ellis 2000; Kaiser, Wilson \& Luppino 2000), and recent measurements are quite impressive in their increased precision (van Waerbeke et al. 2001). However, all measurements to date have been from 


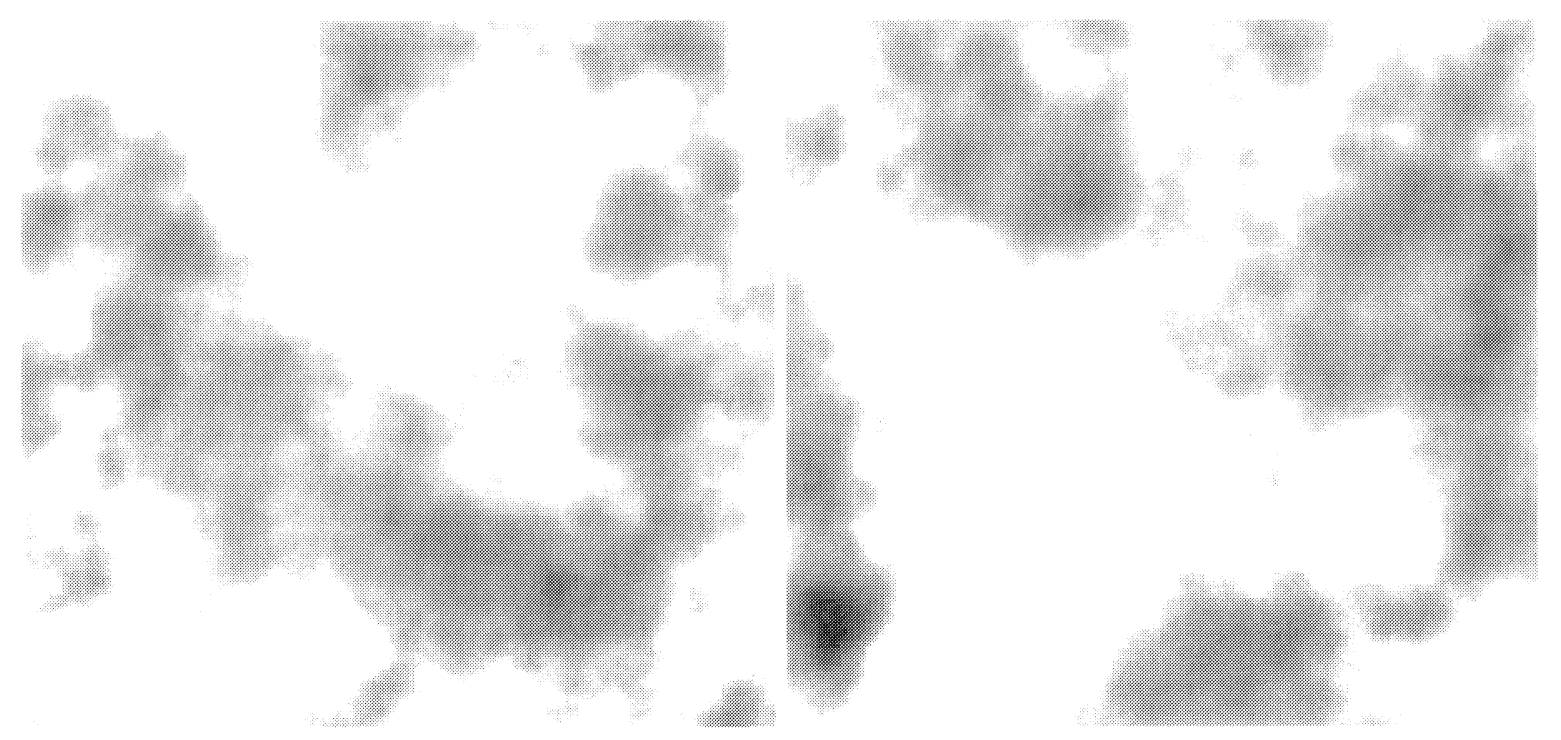

Figure 7. Tomographic view of the W2001 cluster. Darker areas indicate higher density, with most gray being in the noise. No significant structures are seen in the mass map made from sources at $z<0.3$ (left). The cluster appears prominently in the lower left of the mass map made from sources at $z>0.3$ (right). The field is about $40^{\prime}$ on a side. LSST will survey at least three orders of magnitude more area, and enable finer slicing of the redshift distribution, which will provide a detailed picture of the growth of structure with cosmic time.

magnitude-selected samples, which contain galaxies at a large range of redshifts. The use of photometric redshifts would allow separation of sources into redshift bins. Shear correlations as a function of redshift would then trace the evolution of structure over cosmic time, from half the current age of the universe (a rough limit set by the efficiency shown in Figure 2 and by the difficulty of measuring sources at $z>2$ ) to the present. The DLS is currently working on measuring this effect, but for true precision cosmology we need the LSST to expand the area survey by $2-3$ orders of magnitude.

\section{EXTRAPOLATION TO LSST}

The LSST will repeatedly survey large portions of the sky to unprecedented depth. LSST will observe 1000 $\operatorname{deg}^{2}$ in multiple colors, yielding 100 million source galaxies out to $z \sim 3$, giving rise to a mass-selected sample of over 5000 clusters with known redshifts. Hennawi et al. (2002) found that the same analysis applied to the LSST cluster sample will determine $w$ to within a percent, sharply constraining the physical nature of the dark energy.

Hennawi et al. (2002) calculated weak lensing maps from numerical simulations of the cold dark matter (CDM) models with different cosmological parameters. They found that the redshift distribution of clusters detected is very sensitive to $\Omega_{m}$ and $w$. Furthermore, its degeneracies are complementary to that of the CMB, so that a combination of these measurements can firmly break the degeneracies. Huterer (2002) came to similar conclusions about the ability of $\sim 1000 \mathrm{deg}^{2}$ cluster-counting weak lensing surveys to constrain $\Omega_{m}$ and $w$, and the complementarity of that measurement with supernova probes of the expansion history.

Even within the LSST weak lensing dataset, complementary measurements will be made. For example, the change in cosmic shear with source redshift is another way to reveal the evolution of structure. Yet it tests the model in a different way, because it measures the cumulative effect of all structures, not just those above a given threshold. It is also complementary in the sense of having different systematics. With LSST, systematics will become very important as statistical errors shrink dramatically from today's levels. In lensing most systematics come from an anisotropic and inhomogeneous point-spread function, and cosmic shear (correlating all pairs of galaxies) probes these effects in a different way than does cluster detection (tangential shear around a small set of points). 
In summary, the LSST dataset will make it possible to image the mass distribution in three dimensions throughout a large fraction of the universe. This first view of the growth of structure with cosmic time will provide a stringent test of cosmological models and of the nature of dark matter and dark energy.

\section{REFERENCES}

1. Angel, J. R. P., Claver, C. F., Sarlot, R., Martin, H. M., Burge, J. H., Tyson, J. A., Wittman, D., \& Cook, K. American Astronomical Society Meeting, 199, 2001

2. Bacon, D., Refregier, A. \& Ellis, R., MNRAS, 318, 625, 2000

3. Benitez, N., ApJ, 536, 571, 2000

4. Cohen, J. G., Hogg, D. W., Pahre, M. A., Blandford, R., Shopbell, P. L., \& Richberg, K. ApJS, 120, 171, 1999

5. Colless, M. et al., MNRAS, 328, 1039, 2001

6. Connolly, A., et al., AJ, 110, 2655, 1995

7. Erben, T., van Waerbeke, L., Mellier, Y., Schneider, P., Cuillandre, J.-C., Castander, F. J., \& Dantel-Fort, M., $A \& A$ 355, 23, 2000

8. Gray, M. E., Ellis, R. S., Lewis, J. R., McMahon, R. G., \& Firth, A. E., MNRAS, 325, 111, 2001

9. Hennawi, J. F., Narayanan, V. K., Spergel, D. N., Dell'Antonio, I. P., Margoniner, V. E., Tyson, J. A., \& Wittman, D., American Astronomical Society Meeting, 199, 2002

10. Hogg, D., et al., AJ, 115, 1418, 1998

11. Hu, W., PRD, submitted, 2002 (astro-ph/0208093)

12. Hu, W. \& Keeton, C., PRD, submitted, 2002 (astro-ph/0205412)

13. Huterer, D., PRD, 65, 063001, 2002

14. Kaiser, N., Wilson, G. \& Luppino, G., ApJL, submitted, 2000 (astro-ph/0003338)

15. Miralles, J.-M. et al., $A \& A, \mathbf{3 8 8}, 68,2002$

16. Taylor, A., $P R L$, submitted, 2002 (astro-ph/0111605)

17. Tyson, T., Wittman, D., Hennawi, J., \& Spergel, D. 2002, American Physical Society, April Meeting, Jointly Sponsored with the High Energy Astrophysics Division (HEAD) of the American Astronomical Society April 20 - 23, 2002 Albuquerque Convention Center Albuquerque, New Mexico Meeting ID: APR02, abstract \#Y6.004, 6004, 2002

18. Umetsu, K. \& Futamase, T., ApJL, 539, L5, 2000

19. van Waerbeke, L. et al., $A \& A, \mathbf{3 5 8}, 30,2000$

20. van Waerbeke, L. et al., $A \& A, \mathbf{3 7 4}, 757,2001$

21. Wittman, D., Tyson, J. A., Margoniner, V. E., Cohen, J. G., \& Dell'Antonio, I. P., ApJL, 557, L89, 2001

22. Wittman, D. et al., Proc. SPIE, 4836 (these proceedings), 2002a

23. Wittman, D., Margoniner, V. E., Tyson, J. A., Cohen, J. G. \& Dell'Antonio, I., ApJL, submitted, 2002b 